Dermatologische Zeitschrift. 1905;12:I-V

\title{
Contents, Vol. 12, 1905
}

Dermatologisehe Zeitsehrift.

Herausgegeben

von

Prof. Dr. O. LASSAR.

Band XII.

Mit zahlreichen Abbildungen im Text und 21 Tafeln.

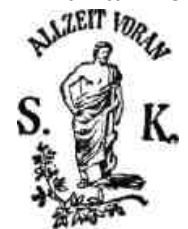

Berlin 1905.

VERLAG VON S. KARGER

KARLSTRASSE 15.

Alle Rechte vorbehalten.

Gedruckt bei Imberg \& Leîson in Berlin W. 9.

Inhalts -Verzeichnis.

Originalarbeiten.

Blanck, Ein aseptisches Taschen-Injektions-Besteck . . 695 Bloch, M., Ein Fall von gonorrhoischer Myelitis . . 443 Bornemann, W., Ueber Besonderheiten beim Lupus erythematosus 349

Bulkley, L. Duncan, Krankheiten der Haut in Verbindung mit den Krankheiten des Stoffwecbsels 522

Delbanco, Ernst, Zur Zungentuberkulose der Papageien

(Hierzu Taf. Ill-IV) . 222

Fürst, L., Die Erzielung von Analgesie auf endermatischem

Wege 756

Galewsky, Ueber Eucalyptus-Dermatitiden ..... 36 Gassmann, A., Ueber einen chronischen pigmentierten

hyperämisch - papulösen Ausschlag (Urticaria pig-

mentosa?) 284

Glaserfeld, Bruno,Welche Beziehungen bestehen zwischen

Haut- und Nierenkrankheiten?

Guszman, Josef, Anatomie der Dermatitis papillaris capil-

litii (Kaposi) (Hierzu Taf. II) 139

Hellev, < i., Beiträge zur Pathologie der spitzen Kondylome 670 Hoffmann, Erich, Ueber eine durch Scilla maritima hervor-

gerufene vesíkulöse Dermatitis nebst Bemerkungen

über die Bedeutung der Raphiden (Hierzu Taf. VI 
bis VII) . . . . 387

- $\quad$ - - Ueber einen Fall von z. T. gangränösen Chancres

mixtes an Lippe und Zunge mit später auftretendem Pseudochancre am Unterarm (Hierzu Taf.

$\mathrm{X}) \ldots 491$

- $\quad$ - - Ueber das Zusammentreffen von Lichen ruber nnd

Diabetes mellitus nebst Mitteilung des histologischen

Befundes bei Lichen sclerosus $\quad 654$

$-\mathrm{IV}$ -

Hübner, Hans, Ueber den jetzigen Stand unserer Kennt-

nisse von der Spirochaete pallida 718

Justus, J., Ueber Arsenvergiftung auf Grund einer mikro-

cheniisch-histologischen Methode (Hierzu Taf. V) . . 277

Kromayer, Rotationsinstruniente 26

v. Marschalko, Th., Ueber einen eigentümlichen Fall zirkumskripter, profuser Hauttalgsekretion (Hierzu

Taf. XX-XXI) 718

Pinkus, Felix, Lichen planus zoniformis 216

v. Poor, Franz, Ueber die linienförmigen Narben bei der

hereditären Syphilis 207

- $\quad$ - - Zur Anatomie der Schleimhautaffektionen bei

Lichen planus (Wilson) 645

Raschkow, Kombination von Psychose und Hautkrankheit 77 Rath, Hugo, Zur Therapie des

Unterschenkelgeschwürs

mit „doppelter Binde” 503

Scherber, G., Zur Röntgenbehandlung der Sycosis simplex

(Hierzu Taf. IX) .435

Schüller, Max, Ueber hereditär - syphilitische Herd-

erkrankungen bei Kindern und die Verbreitung der

protozoischen Parasiten in denselben 1

Selenew, J. F., Exsudationen” und Keratosen. (Hierzu

Tafel XIa-XIX und 1 Kurventafel) 570

Solger, F. B· , Die Bedeutung des Pigments für die hell-

farbigen Menschenrassen 516

Spitzer, Ludwig, Spontane Involution eines Riesennaevus 24 Strebel, H., Ueber Behandlung des Lupus vulgaris der

Haut und Schleimhaut durch hochfrequente Funken-

entladungen 38

- $\quad$ - - Gedankenaustausch mit Professor Niels R. Finsen

über Lichttherapie 115

Suchier, Weitere Mitteilungen über den Wert der stati-

schen Elektrizität für die Behandlung parasitärer

Dermatosen 731

Thimm, P., Oleum cinereum Vasenoli

Tschlenoff, M. A., Ueber die Pagetsche Krankheit (Paget's

disease of the nipple) 91, 237, 293

Vörner, Hans, Beitrag zur Kenntnis des Pigmentes . . 379 
- $\quad$ - - Beitrag zur Kenntnis des Pigments (Hierzu

Tafel XI) 499

- $\quad$ - - Ein Fall von herdförmiger Pigmentanomalie der

Kopfhaare 501

$-\mathrm{V}-$

Vörner, Hans, Fibroma molluscum Virchow 660

-Voss, Thrombophlebitis gonorrhoica 83

- $\quad$ - ßeitrag zur operativen Behandlung des Hirngumma

und der syphilitischen Schädeldachnekrose .... 447 Wechselmann, Wilhelm, Ueber

Pseudoalopecia atrophicans

crustosa (Hierzu Tafel I) 112

Wolters, M., Eigentümliche Veränclerungen in der Haut

über einer Meningocele (Hierzu Tafel VIII) .... 427

Gesellschaftsberichte.

Berliner dermatologische Gesellschaft. Sitzung vom 5. Juli

$1904 \quad 52$

- $\quad$ - - Sitzungen vom 8. November 1904

13. Dezember 1904

313

- $\quad$ - - 10. Janiiar und 14. Februar 1905

461

14. März 1905

552

3. Mai 1905

698

Dänische dermatologische Gesellschaft. Sitzungen vom

4. Februar und 4. März 1903

61

1. April, 7. Oktober u. 4. November 1903 . . 129

2. Dezember 1903, 3. Februar, 2. März, 6. April 1904

Dermatologische und venerologische Gesellschaft zu Charkow.

Sitzung vom 15. April 1903

123

Versammlung deutscher Naturforscher und Arzte in Meran.

Bericht von Priv.-Doz. Dr. Rusch in Innsbruck . . 760

Pepiodisehe Literatup 64,166,293,324,404,598

Besppeehung en 347, 418

Buchanzeigren203, 272, 423, 487, 568, 642

Pepsonalien und Tag· esnaehpiehten . . 76, 138, 348, 426

Ppaktisehe Notizen 76, 206

Zup Syphilis-Statistik ... 275

'Eingabe $\quad 425$ 